\title{
Relationship between pore structure and gas permeability in poplar (Populus deltoides CL.'55/65') tension wood
}

\author{
Yujing Tan ${ }^{1} \cdot$ Jinbo Hu ${ }^{1}$ (D) $\cdot$ Shanshan Chang ${ }^{1} \cdot$ Yuan Wei $^{1} \cdot$ Gonggang Liu ${ }^{1} \cdot$ Qianqian Wang ${ }^{1} \cdot$ Yuan Liu ${ }^{1}$
}

Received: 31 January 2020 / Accepted: 27 August 2020 / Published online: 7 September 2020

(C) The Author(s) 2020

\begin{abstract}
- Key message The important anatomical changes in tension wood, e.g., the high fiber ratio and rich mesopores, did not significantly increase the air and nitrogen flow; thus the gas permeability in the longitudinal direction of poplar (Populus deltoides CL.'55/65') tension wood is actually affected by the cell tissue macroporous porosity.

- Context Gas permeability is one of the most important physical properties of wood and is closely related to its internal microstructure, particularly porosity. Tension wood is widespread in woody plants and displays significant structural differences compared with opposite wood.

- Aims The study was designed to clarify the relationship between pore structure and gas permeability in poplar tension wood. - Methods The gas permeability was measured using a self-made device. The meso- and macroporosity characteristics were measured by nitrogen adsorption-desorption and mercury intrusion porosimetry. The flow was simulated using ANSYS Fluent software to illustrate the role of pore structure on permeability.

- Results The morphological features of vessels have an effect on wood permeability. Compared with tension wood, opposite wood, which has higher vessel ratio, larger cell lumen diameter, and more rich pits, shows stronger gas permeability. Increasing the airflow path will actually reduce the gas permeability. The simulation results are consistent with the experimental results.

- Conclusion In hardwoods, the gas permeability in the longitudinal direction is mainly dictated by the vessels. The high fiber ratio and rich mesopore in tension wood do not significantly increase gas flow, suggesting the permeability of wood was actually determined by the cell tissue with macroporous porosity. Vessel tissue ratio, length and diameter, and intervessel pit size were found responsible for influencing the permeability in the longitudinal direction.
\end{abstract}

Keywords Poplar $\cdot$ Tension wood $\cdot$ Air permeability $\cdot$ Nitrogen permeability $\cdot$ Pore structure $\cdot$ Flow simulation

\section{Introduction}

Wood has a certain level of permeability due to its porous characteristics (Rice and D’Onofrio 1996; Bao et al. 1999; Chun and Ahmed 2006). Permeability reflects the wood's

Handling Editor: Barry A. Gardiner and Patrick Fonti (Guest Editor)

Jinbo $\mathrm{Hu}$

hjb1999@hotmail.com

Shanshan Chang

changelxy@hotmail.com

Yujing Tan

18322597192@163.com

Yuan Wei

wy09aaron@gmail.com ability to be penetrated by gases and liquids and hence is an important physical characteristic. Permeability is also important in the non-mechanical processing of wood as it affects drying and treatment processes such as preservatives and flame retardants and dimensional stabilization processes
Gonggang Liu

liugonggang@gmail.com

Qianqian Wang

csuftqqw@163.com

Yuan Liu

liuyun601220@163.com

1 College of Materials Science and Engineering, Central South University of Forestry and Technology, Changsha 410004, China 
(Wang et al. 1991; Lehringer et al. 2009). The efficiency of gas permeation through wood is closely related to its internal microstructure, particularly porosity, pore size, and connectivity between pores (Lehringer et al. 2009; Ahmed and Chun 2011, 2014). As a natural polymer composite with multiscale hierarchical structure, wood exhibits multiscale pore distributions ranging from the macro to the nano levels. Macroscopic pores such as cell lumen, resin channels, and pit apertures are the main channels for the permeation of liquids (Bao et al. 2001). However, mesoporous pores, such as pores in the cell walls or between cellulose microfibrils, have large specific surface areas and strong adsorption capacities, which are also significant (Choong and Tesoro 1989).

The process of wood formation is often influenced by environmental or other factors (Pilate et al. 2004). In order to adapt to the changes, such as phytohormone, gravity, wind, and other internal and external environment simulation, angiosperms often produce tension wood that generates high tensile stress on the upper side of a leaning stem to maintain the directional growth of the tree (Fisher and Stevenson 1981; Tarmian et al. 2009). In most temperate species, such as poplars, oaks, and chestnuts, tension wood is characterized by the presence of gelatinous (G-) fibers that contain a peculiar cell wall layer called the G-layer (Dadswell and Wardrop 1955; Jourez et al. 2001; Ruelle et al. 2011). The structure of the Glayer is gel-like and contains abundant water-filled mesopores (Clair et al. 2008; Chang et al. 2015, 2017). Significant changes in the cell wall structure of tension wood cause differences in its properties when compared with opposite wood (Pramod et al. 2013). As a 3-D porous material, wood contains natural flow channels (such as vessels). Channels with different diameters, lengths, curves, and microstructures can produce different effects as fluid passes through the wood along the direction of growth.

The gas permeability of wood and its influencing factors have already largely been studied in the literature, but in most cases, it concerned normal wood. The significant anatomical differences especially in pore structure characteristics in tension wood will actually affect its permeability. The main objective of this study is to investigate the relationship between pore structure and gas permeability in tension wood and to clarify the following questions: (i) to what extent does mesopore affect the gas permeability? and (ii) can the flow paths through wood macro-/micropores be traced or simulated? The gas permeability of tension wood was measured by using a self-designed and manufactured experimental device, with special interest on tension wood behavior compared with opposite wood and on the influence of pore structure in its behavior. The mesopore and macropore characteristics of wood samples were analyzed using the nitrogen adsorptiondesorption method and mercury intrusion porosimetry. Current research on the gas permeability in wood is mainly through structural anatomy and observational experiments.
Because of the small size and complex structure of gas conducting tissue in wood, the flow patterns of gas in the wood xylem, is not easily perceived by means of plant physiology. In this study, gas transport at the microscale of wood xylem was studied from the point of view of fluid mechanics. Combined with anatomical observation and experimental research, by means of fluid modeling and numerical simulation, the flow fluid inside the vessels and the influence of vessel structure on flow resistance were simulated using the ANSYS Fluent software which has been already applied to plant xylem flow (Wang et al. 2017; Chen et al. 2017).

\section{Material and methods}

\subsection{Plant material}

A naturally leaning poplar tree (Populus deltoides CL.'55/65') of around $20 \mathrm{~m}$ in height and $23 \mathrm{~cm}$ in diameter at breast height was sampled near the Jiaozuo experimental field in Henan, China. The tree was chosen according to its tilted shape indicating an active process to restore verticality. Poplar was chosen for this investigation as it has previously been used as a model plant for studies on the characteristics of tension wood in several scientific studies (Chang et al. 2014; Roussel and Clair 2015). This species is known to produce tension wood (from the upper side of the inclined axis) with a typical gelatinous layer. The presence of tension wood was confirmed by anatomical observations of a large amount of Gfiber (Vazquez-Cooz and Meyer 2006). The opposite wood from the lower side of the inclined axis was employed as a control.

\subsection{Sample preparation}

Wood blocks were taken from the upper side (tension wood) and the lower side (opposite wood) of the inclined stem. Along the direction of the fiber, blocks were gradually trimmed into cylinders of 8-mm diameter using a sharp blade in wet conditions. Blocks in lengths of 4,6 , and $8 \mathrm{~mm}$ were prepared from the tension and opposite wood. Three samples of each length were made. The remaining fragments that were formed during the trimming of samples were collected and used for pore texture measurements.

\subsection{Optical microscopy}

Transverse sections (10- $\mu \mathrm{m}$ thick) were cut using a sliding microtome (G.S.L.1, Switzerland) with disposable razor blades. Transverse sections were stained with safranin $\mathrm{O}$ /fast green FCF mix and observed under a light microscope (Nikon H550S). This method allows us to identify tension wood and opposite wood quickly. The G-fibers were easily 
distinguished from other fibers after double staining, which resulted in lignified layers being stained red and varying degrees of green, whereas the main cellulosic cell wall layers, e.g., the G-layers, are stained bluish green. On the cross sections, proportions of the various cell types (vessels, fibers, ray, and axial parenchyma) in both tension wood and opposite wood were measured using image analysis software (ImagePro Plus 6.0). Supercritical dried samples were observed using a scanning electron microscope (SEM, Quanta 450) on tangential sections after platinum metallization.

\subsection{Measuring gas permeability}

Permeability measurements in the axial direction were carried out using self-designed and manufactured experimental apparatus (Appendix Fig. 9) (Liu et al. 2019) with air and nitrogen gas as the fluid medium. The devices were designed according to Darcy's law as fluids generally follow this law when passing through wood (Hansmann et al. 2002). Darcy's law states that a linear relationship exists between fluid flux and pressure when fluid flows through wood and other porous solid materials, usually identified by the following formula:

$\mathrm{K}=\frac{F}{\Delta P}$

where $K$ is the permeability coefficient, $F$ is the permeation flux, and $\Delta P$ is the pressure difference.

The air permeability device, as shown in Appendix Fig. 9b, consists mainly of a small air pump, filter, control valve, and flow meter. The device is used by turning on the vacuum pump, slowly adjusting the precision vacuum gage pointer by one grid ( $0.02 \mathrm{MPa}$ is a unit and each unit is divided into 9 grids, i.e., each adjustment of one grid is around $0.0022 \mathrm{MPa}$ ), and recording the number on the digital flowmeter under different pressures. The permeate flux was calculated on the basis of stable permeate volume divided by filtration time, with a unit of $\mathrm{L} / \mathrm{min}$. The nitrogen gas permeability device, as shown in Appendix Fig. 9c, consists mainly of a nitrogen tank, pressure gage, and flow meter. The device is used by opening the valve of the nitrogen tank, slowly adjusting the pointer of the pressure gage, and recording the number on the digital flowmeter under different pressures.

Sample preparation and test conditions were the same for both permeability devices. Supercritical dried cylindrical samples were glued with a modified acrylate adhesive to the end of the matching tube (Appendix Fig. 9a). Modified acrylate adhesive can be used for bonding wood, metal, ceramic, and quartz. The applicable temperature range is from -60 to $100{ }^{\circ} \mathrm{C}$, the curing time at room temperature is $24 \mathrm{~h}$, and the bonding strength can reach $18 \mathrm{MPa}$. To ensure accuracy in the measurements, the samples had to be attached completely to the tube with no gap and contamination of the test surface was avoided throughout the preparation process. The well-adhered samples were then clamped to the device for testing.

In order to check the reproducibility of our measurements, another poplar tree and other five species were also sampled and tested, and the results, which were in good agreement with the data presented in the main body of the article, are shown in Appendix Fig. 10.

\subsection{Permeability simulation of vessel}

In order to clarify the relationship between pore structure and gas permeability and to understand the dynamic transport process of gas in the main flow channel, the fluid distribution in the vessels at a given pressure was simulated using the ANSYS software (Fluent 19.0, ANSYS Inc., Pennsylvania, USA). The ANSYS Fluent is a flow analysis software that can simulate fluid flow, heat transfer, and chemical reactions. It has recently been used to simulate fluid transport in plant xylem (Wang et al. 2017; Chen et al. 2017).

The simulation process can mainly be divided into three steps. Firstly, the basic structural parameter data obtained from the anatomical measurement is applied to the preprocessing module of the software. A single vessel model of tension wood and opposite wood was established and meshed for subsequent calculations. Then, the analysis and calculation module is used to perform the fluid dynamic analysis, and the gas (air or nitrogen) flow characteristics through a single vessel can be obtained. Finally, the simulation results are analyzed and compared using the post-processing module.

\subsection{Measurements of pore texture}

In this study, gas adsorption and mercury intrusion methods were used to characterize the surface area of the wood and pore size distribution. Before testing, all samples were supercritically dried to maintain the integrity of the internal pore structures (Clair et al. 2008). To ensure the consistency, samples were mostly obtained from the fragments remaining from the permeability sample preparation. Samples $(2 \times 2 \times 2 \mathrm{~mm})$ were kept in $30 \%$ ethanol and dehydrated using a gradient of ethanol solutions $[(30,50,70,85,95$, and $100 \%$ (three times)] for $24 \mathrm{~h}$ each. The dehydrated samples were then introduced into a Quorum K850 critical point drier (Quorum Technologies Limited, Kent, UK) with liquid $\mathrm{CO}_{2}$ as the transitional fluid and supercritically dried without surface tension.

Nitrogen adsorption measurements were performed using a Coulter SA3100 surface area analyzer (Beckman Coulter, Inc. Florida Miami, USA) at $77 \mathrm{~K}$. Prior to the adsorption experiment, supercritically dried samples (masses between 0.1 and $0.3 \mathrm{~g}$ were preferable) were outgassed at $75^{\circ} \mathrm{C}$ under a vacuum until the pressure was stabilized at $3 \times 10^{-5} \mathrm{MPa}$ to remove the physically adsorbed gases and impurities from the 
pore surface of the samples. This experimental technique allowed us to characterize the mesoporous texture and record the adsorption-desorption isotherm. The adsorption at low relative pressure allows the specific surface area of the samples to be evaluated by the BET method (Brunauer et al. 1938), assuming an adsorbed $\mathrm{N}_{2}$ molecule covers $0.162 \mathrm{~nm}^{2}$. The method of Broekhoff and de Boer (1968), which make use of the modified Kelvin equation, is used for mesopore size analysis. This method is more suitable for the pore size distribution calculation of wood materials (Clair et al. 2008; Chang et al. 2009).

Mercury intrusion measurements were carried out using a Micromeritics AutoPore IV9510 apparatus (Micromeritics instrument Ltd., Atlanta, USA). The dry sample mass was around 0.1 to $0.2 \mathrm{~g}$. The test procedure was initiated with a low-pressure stage, followed by a high-pressure stage with a maximum intrusion pressure of $414 \mathrm{MPa}$. This technique allows the evaluation of the macropore and/or mesopore size and records the adsorption-desorption isotherm distribution from the incremental volumes of mercury by applying the Washburn equation (Washburn 1921).

\section{Results}

\subsection{Microscopic observation}

Transverse sections stained with safranin/fast green to identify tension wood and opposite wood are illustrated in Fig. 1. Compared with opposite wood, tension wood contained large fibers with well-differentiated G-layers, which were very obviously bluish green after double staining (Fig. 1a). Tension wood is anatomically very different from opposite wood both with respect to the distribution of vessels and fibers. The most notable contrast was that vessels in the opposite wood had relatively larger tangential lumen diameters $(80 \mu \mathrm{m})$ than those in tension wood $(65 \mu \mathrm{m})$. There was also a higher proportion of vessels in opposite wood (34\%) than in tension wood (23\%). Consequently, fibers formed a higher proportion of tension wood (64\%) than opposite wood (52\%). This may be explained by the fact that fibers have a mechanical role in supporting tension wood for orientated growth (Ruelle et al.
2011). No apparent difference was found between the proportions of rays in tension and opposite wood. The value was about $9 \%$ in both.

Observation of tangential sections by SEM showed that plurality of pore structures on the vessel walls could be observed both in tension wood and opposite wood (Fig. 2). There was no apparent difference in the intervascular pit aperture between tension wood and opposite wood, with mean values of between 3 and $6 \mu \mathrm{m}$ (60 measurements). Equally, the pits exhibited similar patterns of distribution in both types of wood.

\subsection{Gas permeability}

In order to validate the stability of the test devices and the repeatability of the measurements, herein, the results of gas permeability measurements in tension wood are shown in Fig. 3, with three replicates of each test (a 6-mm-long tension wood sample was used as an example). The results were consistent and stable, and good reproducibility was also obtained in opposite wood samples of different lengths. Meanwhile, binding permeability test data, $F=\mathrm{a}^{*} P+\mathrm{b}$ ( $F$ for the flux and $P$ for the pressure, $a$ and $b$ are variables), were used to model complete pressure and gas flux in regression analysis (Appendix Table 2). The pressure and flux showed stable linear correlations and the correlation coefficients are between 0.9811 and 0.9991 . These results indicate the high repeatability and stability of measurements taken with our self-designed test device.

Figure 4 shows the air and nitrogen permeability of poplar opposite and tension wood samples with different longitudinal lengths. The gas permeability of opposite wood was higher than that of tension wood samples of the same size. In the two types of wood, the nitrogen and air permeability trends were the same regardless of the sample length and demonstrate that the permeability of nitrogen is higher than that of air.

\subsection{Mesopore structure}

The nitrogen adsorption-desorption isotherms and pore size distribution for poplar opposite and tension wood are shown in Fig. 5. According to the IUPAC classification (Thommes
Fig. 1 Transverse sections of poplar tension wood (a) and opposite wood (b) stained with safranin $\mathrm{O}$ and fast green.

Unlignified G-layer stained in green-blue in tension wood and lignified cell wall layers stained in red in opposite wood and tension wood. Scale bar $(a, b)=30 \mu \mathrm{m}$
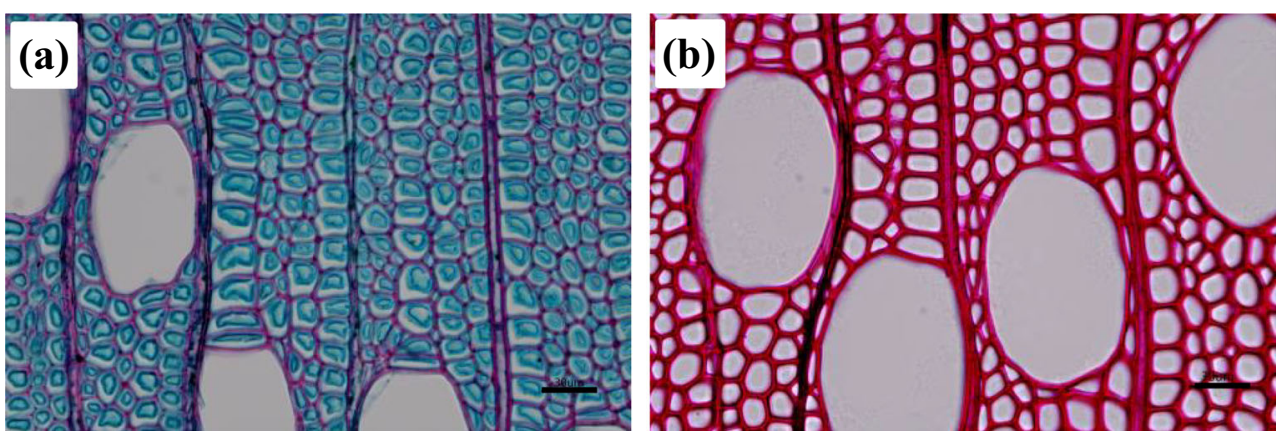
Fig. 2 Ultrastructure of the tangential sections of poplar tension wood (a) and opposite wood (b) after supercritical drying. Scale bar $(a, b)=50 \mu \mathrm{m}$
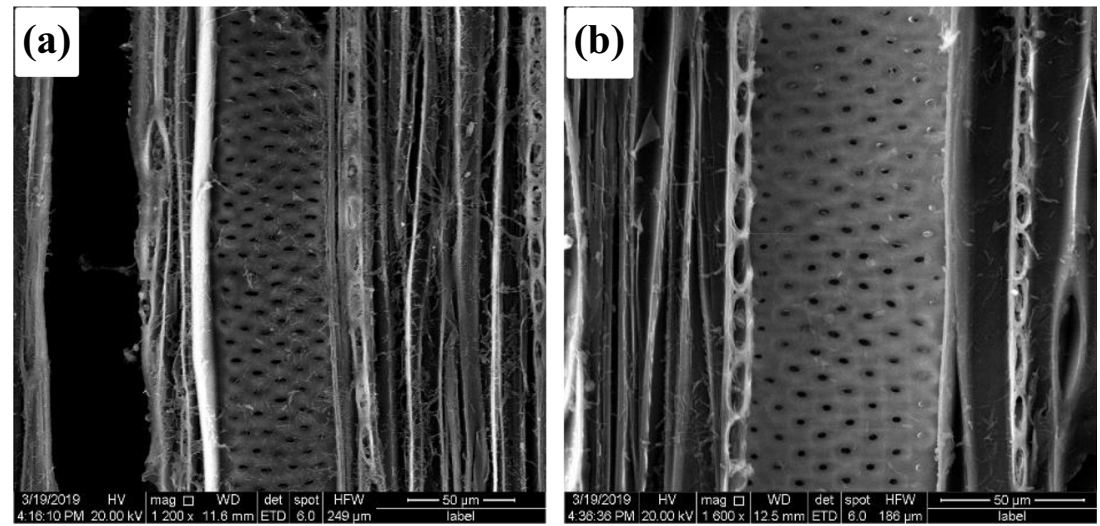

et al. 2015), the isotherm of tension wood (Fig. 5a) belongs to type IV (a), with a H3-type hysteresis loop, indicating the typical presence of mesopores (Kuila and Prasad 2013). The amount of adsorbed nitrogen was 43 times greater in tension wood than in opposite wood, corresponding to a significantly higher mesopore-specific surface area in the tension wood $\left(51.8 \mathrm{~m}^{2} \mathrm{~g}^{-1}\right)$ than in the opposite wood $\left(4.18 \mathrm{~m}^{2} \mathrm{~g}^{-1}\right)$. The specific surface area was strongly correlated with the mesopore volume. In tension wood, the mesopore volume was $0.11 \mathrm{ml} / \mathrm{g}$, accounting for $85 \%$ of the total pore volume, which was seven times higher than that of opposite wood. For opposite wood, the mesopore volume was $0.0158 \mathrm{ml} / \mathrm{g}$, accounting for $74 \%$ of the total pore volume. Both samples presented broad pore size distributions ranging from 3 to $50 \mathrm{~nm}$ (Fig. 5b). In tension wood, $46 \%$ of the mesopores were between 3 and $20 \mathrm{~nm}$ in size, with a peak pore size of $3.7 \mathrm{~nm}$.

\subsection{Macropore structure}

The cumulative pore volumes measured in tension and opposite wood using the mercury intrusion method are shown in Fig. 6a. Under increasing pressure, mercury first filled the macropores and then the micro-sized pores (Zuo and Ye 2018). When filling pores with diameter larger than $75 \mu \mathrm{m}$, the difference in the cumulative pore volume of the two samples was small. Under an increasing mercury pressure, the difference in cumulative pore volume between the two samples became larger, especially in the pores ranging in size from 85 to $38 \mu \mathrm{m}$. The increasing rate of pore volume in opposite wood was greater than that in the tension wood. As pores with diameters less than $38 \mu \mathrm{m}$ were filled gradually, the growth rates for pore volume in the two samples tended to be similar. Figure $6 \mathrm{~b}$ shows the pore size distributions for tension and opposite wood in logarithmic form. Both samples exhibited broad pore size distributions ranging from 0.01 to $334 \mu \mathrm{m}$, with the peak size at $52 \mu \mathrm{m}$. Around $46 \%$ of macropores were distributed between pore sizes of between 30 and $90 \mu \mathrm{m}$, which was the diameter range for the vessel lumens.

Table 1 shows the macroporous structural parameters for poplar tension wood and opposite wood. Compared with tension wood, opposite wood had greater macropore volume and higher porosity. Tension wood possessed more pores less than $30 \mu \mathrm{m}$, whereas opposite wood had a high proportion of pores between 30 and $150 \mu \mathrm{m}$ in diameter, which corresponds to the high vessel ratio and bigger lumen diameter.
Fig. 3 Air (a) and nitrogen (b) permeation flux curves for poplar tension wood with a longitudinal length of $6 \mathrm{~mm}$. Three replicates were carried out in each case and marked as $-1,-2$, and -3 . TW6: tension wood with longitudinal length of $6 \mathrm{~mm}$
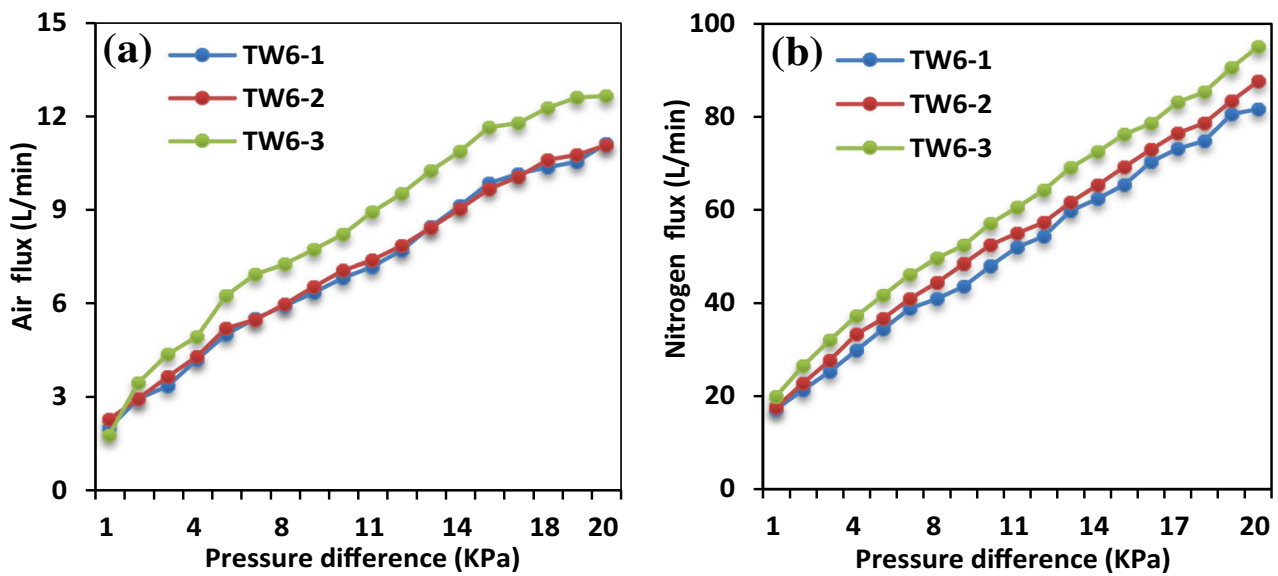


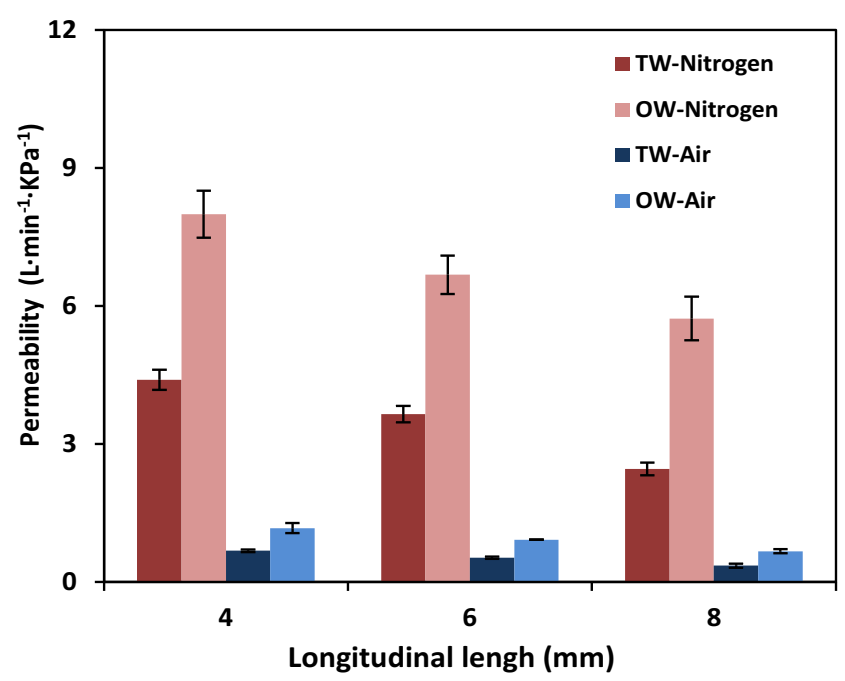

Fig. 4 Comparison of air and nitrogen permeability for poplar opposite wood (OW) and tension wood (TW) samples of different longitudinal lengths. Black bar represents standard deviation (SD)

\subsection{Permeability simulation within vessel}

To better illustrate the difference in gas permeability between tension wood and opposite wood and understand the dynamic transport process of gas in the main channel, the fluid distribution in the vessels at a given pressure was simulated using the ANSYS Fluent software. The morphological sizes of vessel models were based on the measurements of cell segregation and SEM observations (Fig. 7a). Vessels in tension wood and opposite wood were simplified as cylindrical channels with diameters of 65 and $80 \mu \mathrm{m}$ and lengths of 548 and $508 \mu \mathrm{m}$, respectively; additionally, 30 and 50 micropores were distributed in the cell walls, respectively (Fig. 7b). The simplified vessels had micropore aperture diameters of $5 \mu \mathrm{m}$. Data for all geometries were scaled according to actual measurements.
Firstly, the simulation of air passing through the channels with and without micropores in the cell wall was compared (Fig. 8). Significant differences were found to exist in these two types of channels. The flow velocity of the channel with no micropores in the cell wall (Fig. 8b) was low $\left(97 \mathrm{~m} \mathrm{~s}^{-1}\right)$ at the center, whereas the flow velocity for the channel containing micropores in the cell wall (Fig. 8a) was higher at the center $\left(131 \mathrm{~m} \mathrm{~s}^{-1}\right)$. The velocity through the micropore contour lines indicates that the flow velocity weakens from the center to the wall $\left(7 \mathrm{~m} \mathrm{~s}^{-1}\right)$. Some of the gas is diverted through the micropores on the side wall, but the flow velocity is significantly lower than at the center of the vessel.

For poplar, a type of hardwood, gas transport through the wood channels is dictated by the vessels. The gas flow can be changed by changing the corresponding features of the wood channels, e.g., channel diameter and number of vessel pits. Figure $7 \mathrm{c}$ shows the flow velocity contours of air and nitrogen through single channels in opposite and tension wood. Figure $7 \mathrm{~d}$ shows the profile of gas flow velocity at both the inlets and outlets of the channels. From the center of the channel to the wall, the velocity profile magnitude at the inlet is more uniformly distributed than that at the outlet, where the velocity magnitude is obviously higher at the center than at the wall of the channel. Because of the diameter difference in these two channels, the flow velocity profile of tension wood is lower than that of opposite wood both at the inlet and the outlet.

\section{Discussion}

This study indicates the feasibility of rapid measurement of longitudinal gas permeability in poplar tension wood
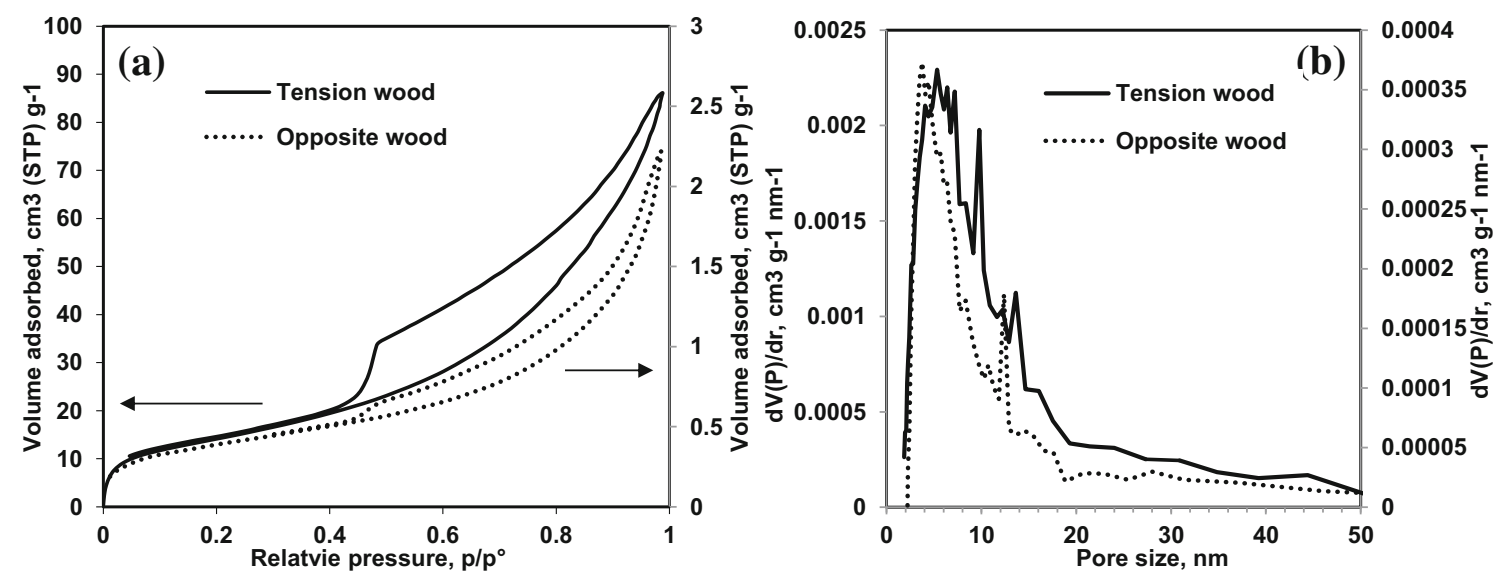

Fig. 5 Nitrogen adsorption-desorption isotherms (a) and pore size distributions (b) in poplar tension wood (solid curve) and opposite wood (dotted curve). The opposite wood data are referred to the secondary axis 

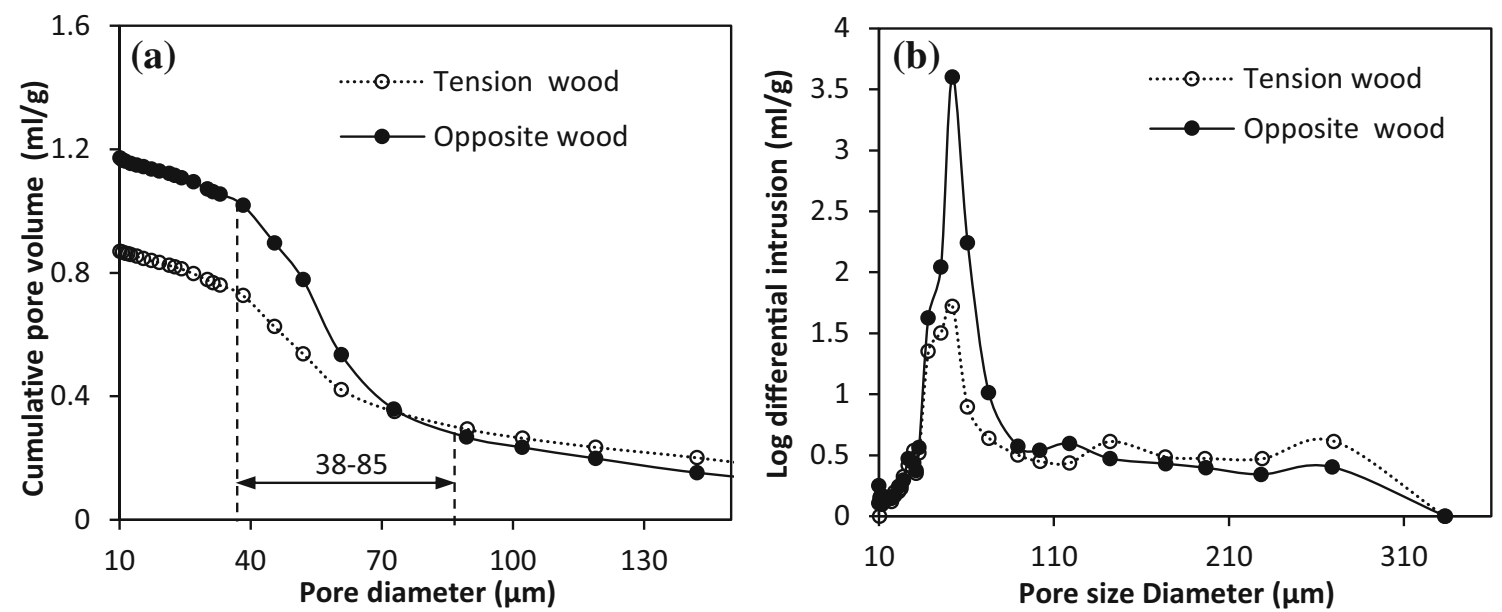

Fig. 6 Mercury intrusion curves of cumulative pore volume (a) and pore size distributions in logarithmic form (b) for poplar tension wood (solid curve) and opposite wood (dotted curve)

at different sample lengths using a self-designed and manufactured experimental device. With the same longitudinal length, poplar opposite wood always showed a higher level of gas permeability than tension wood. The gas permeability difference between these is likely to be related to the internal pore structure, particularly porosity and pore size distribution. As a porous material, wood contains large mixed macro- and micro-capillary systems. The former system consists of the vessel lumen, fibrous cavity, wood ray, intercellular space, etc., and the latter constitutes the pit membrane and pores among the cellulose microfibrils or fibrils in the cell wall. Gas preferentially penetrates wood through the large capillary systems such as the vessel lumen in hardwood species (Greaves 1974). Along the longitudinal direction, the vessel serves as the main transport channel for tree growth and is also the main channel for gas circulation (Leal et al. 2007; Tarmian and Perré 2009). According to its anatomical structure, the arrangement of a vessel is like that of a hollow passage interconnected by perforated plates with adjacent vessels. The prevention of gas flow is, therefore, minimal. Meanwhile, the diameter of the vessel lumen in opposite wood is $15 \mu \mathrm{m}$ larger than that in tension wood, and the vessel ratio is $11 \%$ higher than that in tension wood. Thus, opposite wood provides more favorable conditions for gas flow. Conversely, in tension wood, the tapered ends of fiber channels are interconnected by pits in adjacent cell walls, making it difficult for gas to pass through (Chen et al. 2017). Therefore, the high fiber ratio in tension wood is disadvantageous to gas permeation. Consistent with results obtained for beech tension and normal wood (Tarmian and Perré 2009), increasing the longitudinal length of the sample through which gas passes significantly reduces permeability in both samples. As such, the reduction in specimen length increased permeability. This was thought to be due, in part, to the increased proportion of fibers open at one or both ends of the sample, particularly at lengths below $4 \mathrm{~mm}$. The decrease in permeability at increasing longitudinal lengths indicated either partial or total blockage of some of the conducting pathways as sample lengths exceeded a few cell lengths in hardwoods. Moreover, the significantly lower proportion of narrow vessel lumen and a relatively small number of pits in tension wood is likely to be responsible for the diminished permeability of tension wood in the longitudinal direction (Tarmian et al. 2009). On the other hand, the formation of tension wood is always accompanied by high growth tensile stress. Growth stresses are controlled by the amount of G-layer in tension wood (Fang et al. 2008). Therefore, in tension wood, the characteristic of the inner porous structure will be different under different growth stress and this will lead to the

Table 1 Macroporous structural parameters for poplar tension wood and opposite wood

\begin{tabular}{lllllll}
\hline Pore range & $<30 \mu \mathrm{m} \%$ & $30-90 \mu \mathrm{m} \%$ & $90-150 \mu \mathrm{m} \%$ & $>150 \mu \mathrm{m} \%$ & Porosity $\%$ & Total pore volume (ml g $\left.{ }^{-1}\right)$ \\
\hline Tension wood & 44.27 & 32.81 & 7.98 & 14.94 & 65.1 & 1.29 \\
Opposite wood & 37.69 & 46.08 & 8.21 & 8.02 & 71.3 & 1.73 \\
\hline
\end{tabular}




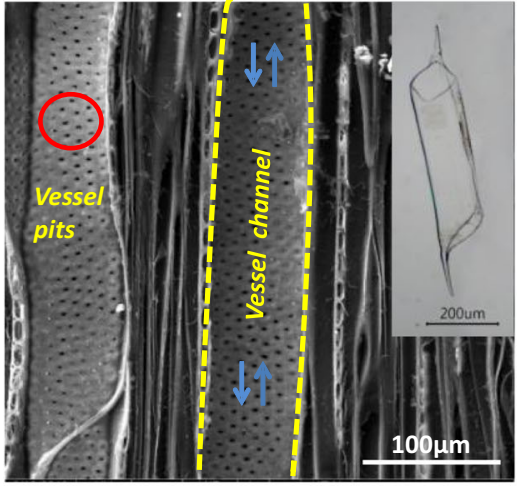

(a)

(b)

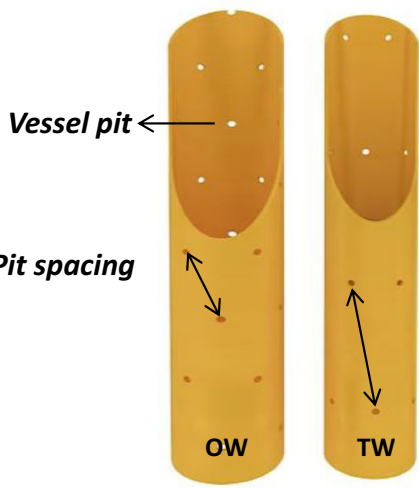

Fig. 7 (a) SEM images showing the channels in the tangential section of poplar opposite wood. The vessel channels are highlighted with yellow dotted lines and the pits on the side wall of the vessel are highlighted with a red circle. Inset is an isolated single vessel. (b) Diagram of vessels in poplar opposite wood (OW) and tension wood (TW) based on wood

differences in wood permeability. The change was due not only to the physical dimensions of the samples but also to a change in the effective pathways within the samples, that is, the effective pore distribution paths.

The nitrogen adsorption-desorption and mercury intrusion measurements showed that poplar opposite wood had an abundance of macropores between 30 and
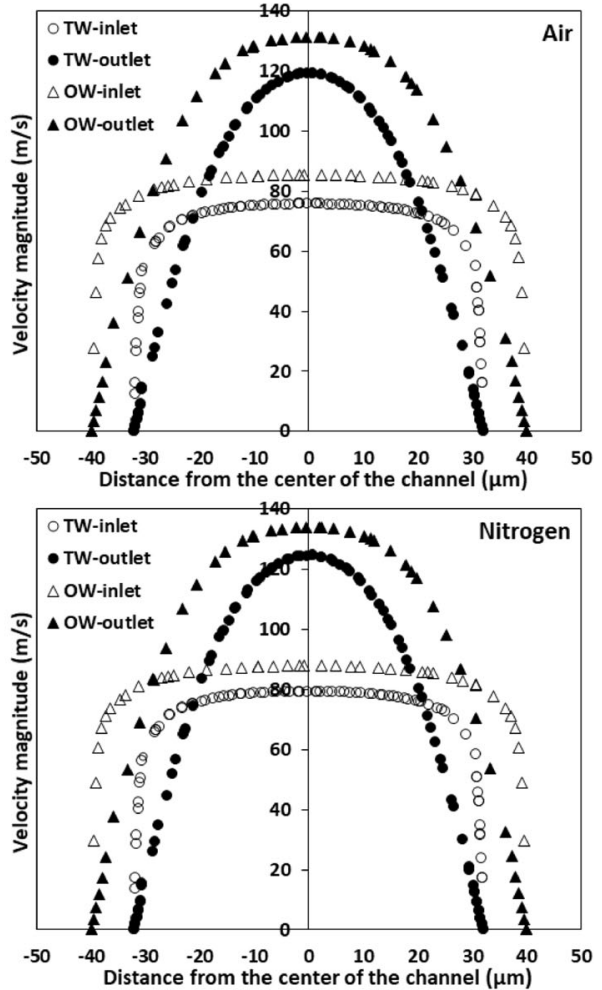

(d)

segregation and SEM measurements. (c) Contours of velocity $\left(\mathrm{m} \mathrm{s}^{-1}\right)$ for air and $\mathrm{N}_{2}$ transport in the vessels of TW and OW based on the Fluent analysis. (d) Air and $\mathrm{N}_{2}$ velocity profiles for TW and $\mathrm{OW}$ at different locations (inlet and outlet) based on the Fluent analysis

$150 \mu \mathrm{m}$ in diameter; however, tension wood had a large number of mesopores between 2 and $20 \mathrm{~nm}$ in diameter, which were confirmed to be present in the G-layer (Clair et al. 2008; Chang et al. 2017). The abundance of mesopores in the G-layers reduced the flow rate for gas in the macro-sized pores, consequently lowering gas permeability. What is more, the mesopores in tension

Fig. 8 Flow velocity contours of air transport at the outlet in cylindrical channels with (a) and without (b) micropores in the cell wall
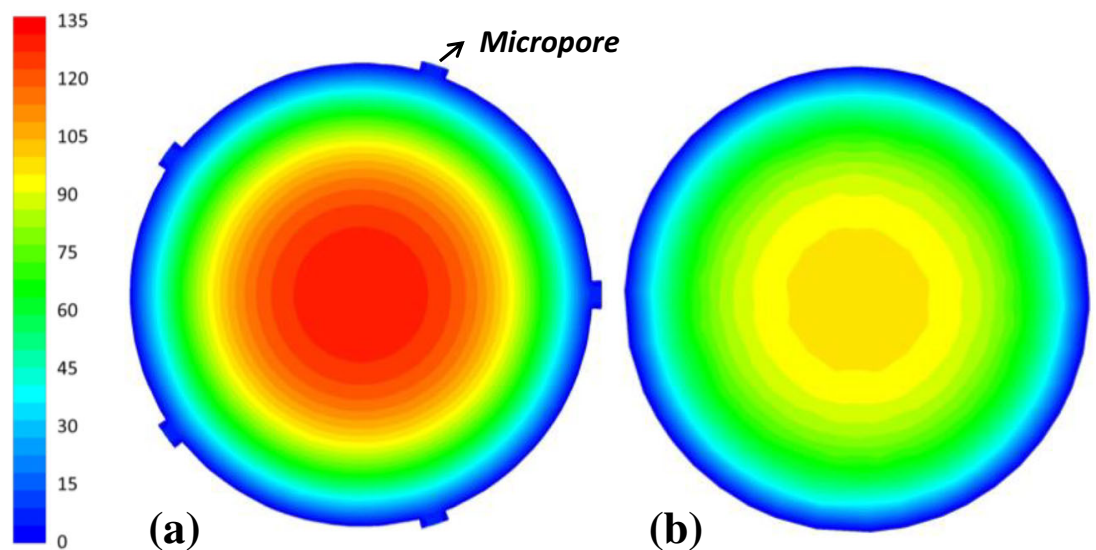
wood showed an ink bottle shape with pore cavities that were less than twice the diameter of the throats, which were beneficial to the adsorption and collection of gas but not conducive to gas permeation (Thommes et al. 2015). At the same time, in tension wood, the relatively narrow lumen diameter increases the contact time between the gas and the channel wall, which enhances the flow of gas closer to the wall, since the micropores in the wall enable gas transport between channels.

Regardless of the wood type, the Fluent simulation results showed that the flow velocity of nitrogen through a single channel is slightly greater than that of air. The tendency is similar in both simulation results and experimental results, but the flow velocity difference between nitrogen and air was obviously reduced in the simulation results. In fact, in the actual experimental measurement, there is a certain amount of water vapor in the air. When the gas passed through the dried wood, water adsorption was inevitable, which reduced the effective gas channels and increased the gas flux difference between air and nitrogen. Compared with the actual experimental environment, the setting of simulation conditions was relatively simple. Firstly, in permeability simulation, this effect of moisture in the air on gas permeability was not fully taken into account. Secondly, only the permeability of a single vessel (the macropore) was simulated; however, the actual permeability channels in wood include not only the macropores but also a large number of mesopores. As a natural porous material, wood contains a complex pore structure system, while the simulation of a single vessel was a relatively ideal state. At the same time, the permeability difference between these two gases could also be attributed to the natural characteristics of the gases themselves. Nitrogen is relatively less dense than air; meanwhile, the friction between nitrogen and wood is less than that for air, resulting in the easier passage of nitrogen through the channel with a relatively higher flow velocity than that of air (Zhao et al. 2006).

The simulation above confirmed that the morphological features of vessels affect wood permeability. Generally, opposite wood has a higher vessel ratio and larger cell lumen diameter than those of tension wood, which shows greater gas permeability. Compared with tension wood, opposite wood contains more pits in the cell wall and these pits are favorable for gas permeation between channels, which is beneficial for gas permeability. In tension wood, the high ratio of fiber content and the abundance of mesopores on the G-layer do not significantly increase gas flow; on the contrary, ink bottleshaped mesopores increase the friction between the gas and wood and block the flow. The simulation results confirmed that the flow velocity at the channel wall decreases significantly at the outlet of the channel (Thommes et al. 2015; Sawada et al. 2018). The simulation suggested that opposite wood, with higher macroporous porosity, has advantages in longitudinal permeability, which was consistent with the experimental results. Meanwhile, increasing the flow passage, i.e., increasing the specimen length, would inevitably reduce gas permeability.

\section{Conclusions}

This study investigated nitrogen and air permeability between poplar tension wood and opposite wood as well as the relationship with its porosity characteristics. Regardless of the wood type, the flow velocity of nitrogen through wood channels is slightly greater than that of air. For hardwoods, the gas transport through the wood channels is mainly dictated by the vessels, that is, the macropore. The abundance of mesopores in tension wood do not significantly increase gas flow. The gas permeability in tension wood has a remarkable dependence on the wood structure, e.g., the channel diameter, the number of vessel pits, as well as the physical dimension of the sample. This study provides a new insight by using fluid flow software to demonstrate gas flow through a single vessel and provides a reference for the simulation of the flow of fluid through wood.

Acknowledgments The author thanks the Jiaozuo experimental field in Henan, China, for providing the wood samples.

Contributions of the co-authors Yujing Tan performed the experiment and wrote the manuscript. Jinbo Hu conceived and designed the experiments and revised the manuscript. Shanshan Chang designed the study and commented on and revised the manuscript. Yuan Wei collected the data and developed the simulation. Gonggang Liu designed the experiments and revised the manuscript. Qianqian Wang performed the experiment and collected the data. Yuan Liu supervised the work and commented on the manuscript. Jinbo Hu and Shanshan Chang contributed equally. All authors reviewed and approved the final manuscript.

Funding This study was funded by the National Key Research and Development Program of China (2017YFD0600202), the Hunan Provincial Natural Science Foundation of China (2020JJ2058), Scientific Research Project of Hunan Education Department (19A505), and Project of Scientific Research Plan of Changsha City (kq1706075).

Data availability The datasets generated during the current study are available from the corresponding authors on reasonable request.

\section{Compliance with ethical standards}

Conflicts of interest The authors declare that they have no conflict of interest. 


\section{Appendix}

(a)

sample

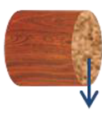

$\underset{\text { Adhesive sealing }}{\stackrel{\text { side }}{ }}$

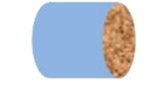

measured direction $(D=8 \mathrm{~mm})$

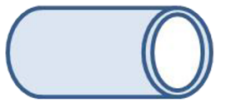

$\underset{\text { adhesive sealing }}{\stackrel{\text { interior wall }}{\longrightarrow}}$
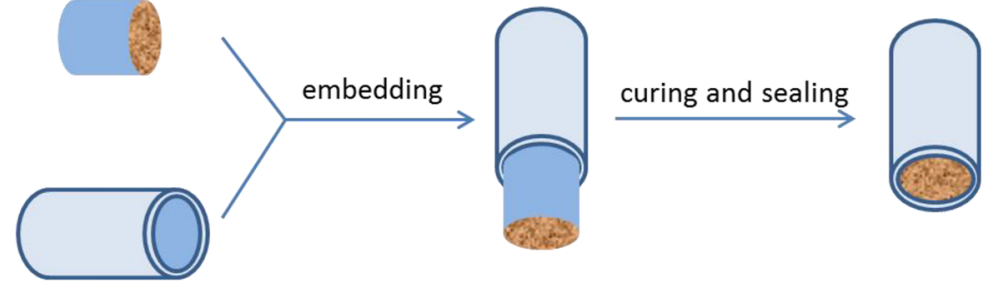

Tefion pipeline $(I D=8 \mathrm{~mm})$

(b) digital flowmeter

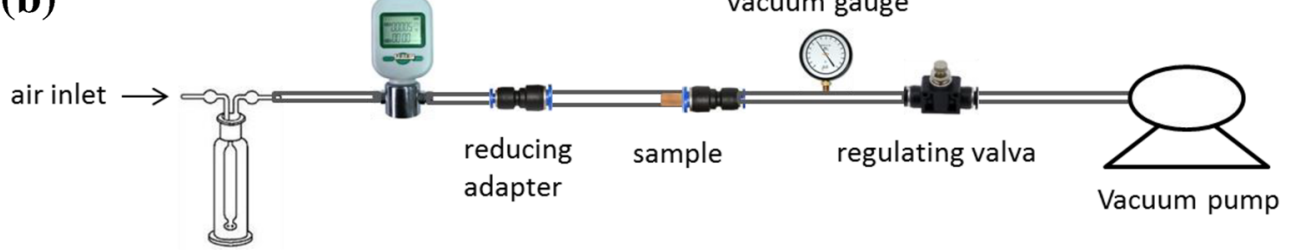

porous gas washing bottle

(c)

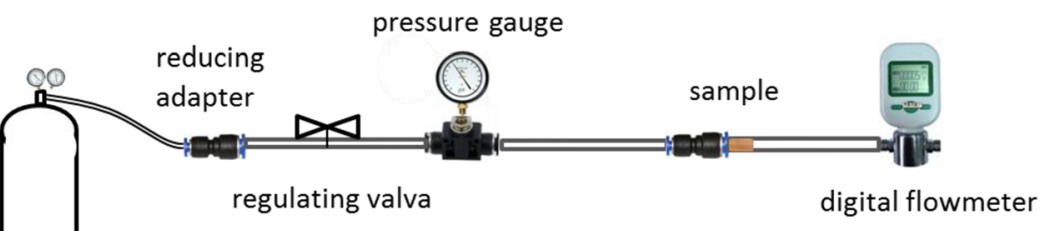

Nitrogen tank

Fig. 9 (a) The process of encapsulating a wood sample in a Teflon pipeline with adhesive sealing. (b) Schematic diagram of the homemade air permeability measuring device. (c) Schematic diagram of the homemade nitrogen permeability measuring device. (ID: internal diameter)

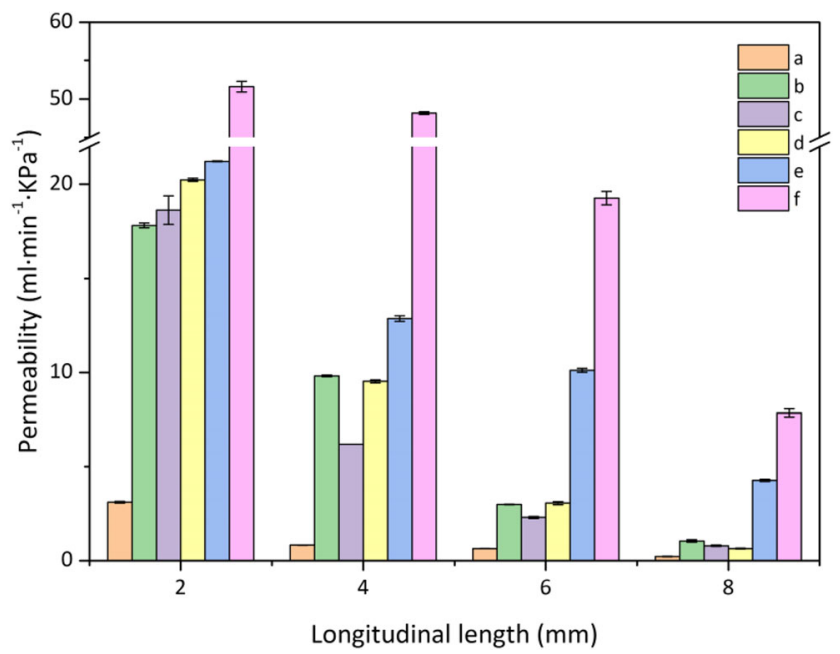

Fig. 10 The air permeability of six wooden samples (a, b, c, d, e, and f) with longitudinal lengths of 2, 4, 6, and $8 \mathrm{~mm}$. (a) Moso bamboo (Phyllostachys edulis). (b) Poplar (Populus euramericana cv. "Zhonglin46"). (c) Chinese fir (Cunninghamia lanceolata). (d) Lophira alata. (e) Beech (Fagus sylvatica). (f) Okan (Cylicodiscus gabunensis). Black bars represent standard deviation (SD) 
Table 2 Correlation analysis between pressure and gas flux in poplar tension wood and opposite wood

\begin{tabular}{|c|c|c|c|c|c|}
\hline & \multirow{2}{*}{$\begin{array}{l}\text { Length } \\
(\mathrm{mm})\end{array}$} & \multicolumn{2}{|l|}{ Air } & \multicolumn{2}{|l|}{ Nitrogen } \\
\hline & & Regression equation & $R^{2}$ & Regression equation & $R^{2}$ \\
\hline \multirow[t]{3}{*}{ Tension wood } & 4 & $F=0.679 P+3.2547$ & 0.9811 & $F=4.3975 P+19.629$ & 0.9972 \\
\hline & 6 & $F=0.5273 P+2.0239$ & 0.9896 & $F=3.6503 P+16.282$ & 0.9972 \\
\hline & 8 & $F=0.3541 P+1.2386$ & 0.9877 & $F=2.4575 P+9.1414$ & 0.9991 \\
\hline \multirow[t]{3}{*}{ Opposite wood } & 4 & $F=1.1706 P+4.3463$ & 0.9980 & $F=7.9973 P+43.141$ & 0.9918 \\
\hline & 6 & $F=0.9206 P+3.1862$ & 0.9983 & $F=6.6818 P+28.634$ & 0.9987 \\
\hline & 8 & $F=0.6683 P+2.9646$ & 0.9970 & $F=5.7286 P+21.722$ & 0.9979 \\
\hline
\end{tabular}

$F$ gas flux $(\mathrm{L} / \mathrm{min}), P$ pressure $(\mathrm{kPa})$
Open Access This article is licensed under a Creative Commons Attribution 4.0 International License, which permits use, sharing, adaptation, distribution and reproduction in any medium or format, as long as you give appropriate credit to the original author(s) and the source, provide a link to the Creative Commons licence, and indicate if changes were made. The images or other third party material in this article are included in the article's Creative Commons licence, unless indicated otherwise in a credit line to the material. If material is not included in the article's Creative Commons licence and your intended use is not permitted by statutory regulation or exceeds the permitted use, you will need to obtain permission directly from the copyright holder. To view a copy of this licence, visit http://creativecommons.org/licenses/by/4.0/.

\section{References}

Ahmed SA, Chun SK (2011) Permeability of Tectona grandis L. as affected by wood structure. Wood Sci Technol 45:487-500

Ahmed SA, Chun SK (2014) Observation of liquid permeability related to anatomical characteristics in Samanea saman. Turk J Agric For $33: 155-163$

Bao FC, Lu JX, Avramidis S (1999) On the permeability of main wood species in China. Holzforschung 53:350-354

Bao FC, Lu JX, Zhao YK (2001) Effect of bordered pit torus position on permeability in Chinese yezo spruce. Wood Fiber Sci 33:193-199

Broekhoff JCP, de Boer JH (1968) Studies on pore systems in catalysts: xii. Pore distributions from the desorption branch of a nitrogen sorption isotherm in the case of cylindrical pores A. An analysis of the capillary evaporation process. J Catal 10:368-374

Brunauer S, Emmett PH, Teller E (1938) Adsorption of gases in multimolecular layers. J Am Chen Soc 60:309-319

Chang SS, Clair B, Ruelle J, Beauchêne J, Di Renzo F, Quignard F, Zhao GJ, Yamamoto H, Gril J (2009) Mesoporosity as a new parameter for understanding tension stress generation in trees. J Exp Bot 60: 3023-3030

Chang SS, Quignard F, Alméras T, Clair B (2015) Mesoporosity changes from cambium to mature tension wood: a new step toward the understanding of maturation stress generation in trees. New Phytol 205:1277-1287

Chang SS, Quignard F, Clair B (2017) The effect of sectioning and ultrasonication on the mesoporosity of poplar tension wood. Wood Sci Technol 51:507-516

Chang SS, Salmén L, Olsson AM, Clair B (2014) Deposition and organisation of cell wall polymers during maturation of poplar tension wood by FTIR microspectroscopy. Planta 239:243-254
Chen F, Gong AS, Zhu M, Chen G, Lacey SD, Jiang F, Li Y, Wang Y, Dai J, Yao Y, Song J, Liu B, Fu K, Das S, Hu L (2017) Mesoporous, three-dimensional wood membrane decorated with nanoparticles for highly efficient water treatment. ACS Nano 11:4275-4282

Choong ET, Tesoro FO (1989) Relationship of capillary pressure and water saturation in wood. Wood Sci Technol 23:139-150

Chun SK, Ahmed SA (2006) Permeability and meniscus phenomenon in four Korean softwood species. Forestry Studies in China 8:56-60

Clair B, Gril J, Di Renzo F, Yamamoto H, Quignard F (2008) Characterization of a gel in the cell wall to elucidate the paradoxical shrinkage of tension wood. Biomacromolecules 9:494-498

Dadswell HE, Wardrop AB (1955) The structure and properties of tension wood. Holzforschung 9:97-104

Fang CH, Clair B, Gril J, Liu SQ (2008) Growth stresses are highly controlled by the amount of G-layer in poplar tension wood. IAWA J 29:237-246

Fisher JB, Stevenson JW (1981) Occurrence of reaction wood in branches of dicotyledons and its role in tree architecture. Bot Gaz 142:82-95

Greaves H (1974) A review of the influence of structural anatomy on liquid penetration into hardwoods. J Inst Wood Sci 6:37-40

Hansmann C, Gindl W, Wimmer R, Teischinger A (2002) Permeability of wood - a review. Wood Res-Slovakia 47:1-16

Jourez B, Riboux A, Leclercq A (2001) Anatomical characteristics of tension wood and opposite wood in young inclined stems of poplar (Populus euramericana $\mathrm{cv}$ 'Ghoy'). IAWA J 22:133-157

Kuila U, Prasad M (2013) Specific surface area and pore-size distribution in clays and shales. Geoohys Prospect 61:341-362

Leal S, Sousa VB, Pereira H (2007) Radial variation of vessel size and distribution in cork oak wood (Quercus suber L). Wood Sci Technol 41:339-350

Lehringer C, Richter K, Schwarze FWMR, Militz H (2009) A review on promising approaches for liquid permeability improvement in softwoods. Wood Fiber Sci 41:373-385

Liu G, Chen D, Liu R, Yu Z, Jiang J, Liu Y, Hu J, Chang S (2019) Antifouling wood matrix with natural water transfer and microreaction channels for water treatment. ACS Sustain Chem Eng 7:6782-6791

Pilate G, Déjardin A, Laurans F, Leplé JC (2004) Tension wood as a model for functional genomics of wood formation. New Phytol 164:63-72

Pramod S, Rao KS, Sundberg A (2013) Structural, histochemical and chemical characterization of normal, tension and opposite wood of Subabul (Leucaena leucocephala (lam.) De wit.). Wood Sci Technol 47:777-796

Rice RW, D'onofrio M (1996) Longitudinal gas permeability measurements from eastern white pine, red spruce and balsam fir. Wood Fiber Sci 28:301-308 
Roussel JR, Clair B (2015) Evidence of the late lignification of the Glayer in Simarouba tension wood, to assist understanding how nonG-layer species produce tensile stress. Tree Physiol 35:1366-1377

Ruelle J, Beauchêne J, Yamamoto H, Thibaut B (2011) Variations in physical and mechanical properties between tension and opposite wood from three tropical rainforest species. Wood Sci Technol 45: 339-357

Sawada D, Kalluri UC, O’Neill H, Urban V, Langan P, Davison B, Pingali SV (2018) Tension wood structure and morphology conducive for better enzymatic digestion. Biotechnol Biofuels 11:44

Tarmian A, Perré P (2009) Air permeability in longitudinal and radial directions of compression wood of Picea abies L. and tension wood of Fagus sylvatica L. Holzforschung 63:352-356

Tarmian A, Remond R, Faezipour M, Karimi A, Perré P (2009) Reaction wood drying kinetics: tension wood in Fagus sylvatica and compression wood in Picea abies. Wood Sci Technol 43:113-130

Thommes M, Kaneko K, Neimark AV, Olivier JP, Rodriguez-Reinoso F, Rouquerol J, Sing KSW (2015) Physisorption of gases, with special reference to the evaluation of surface area and pore size distribution (IUPAC technical report). Pure Appl Chem 87:1051-1069
Vazquez-Cooz I, Meyer RW (2006) Cutting forces for tension and normal wood of maple. Forest Prod J 56:26-34

Wang J, Dai C, Liu Y (1991) Wood permeability. J Forestry R 2:91-97

Wang Y, Sun G, Dai J, Chen G, Morgensten J, Wang Y, Kang S, Zhu M, Das S, Cui L, Hu L (2017) A high-performance, low-tortuosity wood-carbon monolith reactor. Adv Mater 29:1604257

Washburn EW (1921) The dynamics of capillary flow. Phys Rev Lett 17(3):273-283

Zhao W, He N, Li L (2006) Friction and wear properties of WC-Co cemented carbide sliding against Ti6Al4V alloy in nitrogen gas. Tribol T 26:439-442

Zuo Y, Ye G (2018) Pore structure characterization of sodium hydroxide activated slag using mercury intrusion porosimetry, nitrogen adsorption, and image analysis. Mater 11:1035

Publisher's note Springer Nature remains neutral with regard to jurisdictional claims in published maps and institutional affiliations. 